\title{
Association Between Tumor Budding Grade and T Stage in Predicting Recurrence in High-risk Stage li Colorectal Cancer
}

Hiroyuki Kodama

Osaka Medical College

Toshifumi Yamaguchi ( $\nabla$ yamagu.toshifumi@gmail.com )

Osaka University

Yasunobu Ishizuka

Osaka Medical College

Masahiko Aoki

Osaka Medical College

Takahiro Miyamoto

Osaka Medical College

Tetsuji Terazawa

Osaka Medical College

Fukutaro Shimamoto

Osaka Medical College

Masahiro Goto

Osaka Medical College

Hiroki Hamamoto

Osaka Medical College

Wataru Osumi

Osaka Medical College

Masashi Yamamoto

Osaka Medical College

Keitaro Tanaka

Osaka Medical College

Junji Okuda

Osaka Medical College

Kazuhisa Uchiyama

Osaka Medical College

Kazuhide Higuchi

Osaka Medical College

Hiroki Yukami 


\section{Research Article}

Keywords: pathological stage (pStage) II, recurrence-free survival (RFS), colon cancer, high-risk features (HRF)

Posted Date: March 8th, 2021

DOl: https://doi.org/10.21203/rs.3.rs-284704/v1

License: (c) (1) This work is licensed under a Creative Commons Attribution 4.0 International License. Read Full License 


\section{Abstract}

In pathological stage (pStage) II colon cancer, factors such as $T$ stage $(T)$ are high-risk features (HRF) for recurrence. The SACURA trial showed that tumor budding (BD) grade was also associated with recurrence: the 5-year recurrence-free survival (RFS) rate was lower in patients in the BD3 group compared with that of other groups. Interestingly, the BD3 group had a higher proportion of patients with T4. We investigated the prognostic association between T4 and BD3 for recurrence in pStage II colorectal cancer (pll-CRC) with HRF. We analyzed pll-CRC patients with HRF between 2013-2018 at our hospital, Japan. Inclusion criteria were as follows: $\geq 1$ HRF $[<12$ lymph nodes examined $(<12 \mathrm{LN})$, lymph/vascular-invasion, perineural-invasion, $\mathrm{T}, \mathrm{BD}$, and histologic-type]. We primarily analyzed the relationship between each factor and RFS. Among 2,920 pll-CRC patients, 448 had HRF. Of these, 43 (9.6\%) had T4 and 236 (52.7\%) had BD3. On initial analysis, $<12 \mathrm{LN}(P=0.0412)$, tumor location $(P=0.0023), \mathrm{T} 4(P=0.0023)$, and BD3 $(P=0.0396)$ were independent prognostic factors for RFS. Among 257 patients with BD3 and/or T4, the 5year RFS rates were $81.3 \%, 74.6 \%$, and $66.2 \%$ for patients with BD3 (214 patients), T4 (22 patients), and BD3 plus T4 (21 patients), respectively (hazard ratio $3.08, P=0.0079$ ). pll-CRC patients with BD3 plus T4 had poorer prognosis than those with other factors.

\section{Introduction}

Colorectal cancer (CRC) has been the most common cancer in Japan since 2015, and one-third of patients with curatively resected CRC have stage II disease. ${ }^{1}$ Although adjuvant chemotherapy is the internationally accepted standard treatment for patients with stage III CRC, its usefulness for stage II of this disease remains controversial. The major Western international clinical guidelines suggest that postoperative adjuvant chemotherapy should be considered in patients with stage II colon cancer with at least one of the following high-risk factors for recurrence: inadequate lymph node sampling, obstruction, tumor perforation, poorly or undifferentiated histologic type, lymph or vascular invasion, perineural invasion, and T4 stage. ${ }^{2-4}$ Among these factors, the T4 stage has been suggested to have a stronger relationship with recurrence with reproducibility in some previous studies. For example, Quah et al. demonstrated T4 stage as the highest hazard for survival (hazard ratio [HR] 2.7, 95\% confidence interval [Cl] 1.1-6.2), and Babcock et al. showed its strongest negative impact on survival ( $\mathrm{HR} 2.56,95 \% \mathrm{Cl} 2.03-$ 3.21) in high-risk stage II colon cancer. ${ }^{5,6}$

Tumor budding (BD) grade has been defined as a single tumor cell or small cluster of four or fewer tumor cells at the invasive front ${ }^{7-8}$ and is expected to be a potential poor prognostic factor in stage II CRC. ${ }^{9}$ In 2016, the International Tumor Budding Consensus Conference (ITBCC) reached a consensus on an international, evidence-based, standardized scoring system for BD in CRC. ${ }^{10}$ In 2017, the Union for International Cancer Control (UICC) publication of the TNM Classification of Malignant Tumors ( $8^{\text {th }}$ edition) adopted $\mathrm{BD}$ as a potential tumor-related prognostic factor for recurrence. ${ }^{11}$

The SACURA trial showed no superiority of the 1-year UFT adjuvant chemotherapy over surgery for Stage II colon cancer, although the recurrence rate was lower in the UFT group than in the surgery alone group 
(10.4 vs. $13.4 \%) .{ }^{12}$ Subgroup analysis in the SACURA trial demonstrated that the BD grade, which was classified as BD1, BD2, and BD3 according to the number of budding foci was associated with a high risk of recurrence; the 5-year recurrence-free survival (RFS) rate in the BD3 group (74.4\%) was lower than those in the BD1 (90.9\%) or BD2 (85.1\%) groups. ${ }^{13}$ However, there were more patients who had T4 stage in the BD3 group (T4: 26.8\%) than in the BD1 (T4: 10.4\%) or BD2 (T4: 16.0\%) groups in the SACURA trial. Thus, the RFS in the BD3 group might be strongly affected by the T stage.

The aim of this retrospective study was to investigate the relationship between T4 stage and BD3 as prognostic factors for RFS in high-risk pathological stage (pStage) II CRC.

\section{Methods}

\section{Patients and methods}

We retrospectively reviewed the medical records of CRC patients who were diagnosed with pStage II at Osaka Medical College Hospital, Osaka, Japan, between January 2013 and December 2018. Patients with one or more high-risk features (HRF) examined, including number of lymph nodes ( $\leq 12$ or $>12$ ), histologic type of poorly differentiated or mucinous adenocarcinoma (por or muc), BD grade (BD1, BD2, or BD3), depth of invasion (T3 or T4), lymph or venous invasion, and perineural invasion were enrolled. Patients who were administered neoadjuvant chemotherapy or radiotherapy were also included. As adjuvant chemotherapy, a fluoropyrimidine-based regimen was administered to selected patients based on the physician's decision. The TNM classification was categorized according to the $8^{\text {th }}$ edition of the UICC classification. ${ }^{25}$ As an initial analysis, we evaluated the relationship between each HRF and RFS to confirm the risk factors for recurrence in high-risk pStage II CRC. Second, we analyzed the RFS for pStage II CRC patients who had T4 and/or BD3 as the primary analysis. The institutional review board of Osaka Medical College Hospital approved this study protocol (no. 2927).

\section{Assessment of BD grade}

The BD grade was evaluated using hematoxylin-and-eosin-stained slides and was assessed in one hotspot (in a field measuring $0.785 \mathrm{~mm}^{2}$ ) at the invasive front. We classified tumors with $<5,5-9$, and 10 or more budding foci as grades BD1, BD2, and BD3, respectively. These assessment criteria were subsequently adopted in the Japanese guidelines ${ }^{26}$ and international criteria in the ITBCC2016. ${ }^{10}$

\section{Statistical analysis}

Univariate and multivariate analyses were performed using Cox proportional hazards regression to identify independent significant risk factors affecting RFS. The associations between each clinicopathological characteristic and RFS in three groups among the combination of BD3 and T4 stage were analyzed using the Chi square test. Survival analysis was conducted using the Kaplan-Meier method, and differences were evaluated using the log-rank test. RFS was defined as the time from surgery to the date of recurrence or death from any cause. The overall survival (OS) was calculated from 
the time of surgery to death from any cause. A two-sided $P$ value $<0.05$ was considered statistically significant. All statistical analyses were performed using JMP Pro, version 15.

\section{Results}

Patient clinical and pathological characteristics in high-risk pStage II CRC

Among the 2920 patients registered, 448 were identified as eligible. Figure 1 presents a CONSORT diagram of the study population. The clinicopathological characteristics of 448 patients with high-risk stage II CRC are shown in Table 1. The median age was 70 (range, 26-88) years. There were 260 (58.0\%) men and $188(42.0 \%)$ women. The tumor was located in the rectum in $188(42.0 \%)$ patients. The depth of invasion was T4 in 43 (9.6\%) patients. The BD grade was BD1 in 122 (27.2\%), BD2 in 70 (15.6\%), and BD3 in $236(52.7 \%)$ patients. Thirty-nine (8.7\%) patients were treated with chemoradiotherapy and 36 $(8.0 \%)$ with chemotherapy before surgery. Fifty-six (12.5\%) patients were administered adjuvant chemotherapy.

The median follow-up time was 46.3 months (range, 0.79-88.8). The 5-year RFS and 5-year OS rates in 448 patients were $83.0 \%$ (95\% $\mathrm{Cl} 78.8-86.6 \%)$ and 93.7\% (95\% Cl 90.2-96.1\%), respectively. Forty-seven $(10.5 \%)$ patients had tumor recurrence: 16 with liver metastasis, 15 with lung metastasis, 10 with local recurrence at the primary tumor site, 7 with lymph node metastasis, 5 with peritoneal carcinomatosis, 2 with brain metastasis, 1 with bone metastasis, and 1 with ovarian metastasis.

\section{Initial analysis to confirm each prognostic factor for recurrence}

Univariate and multivariate analyses to identify significant risk factors related to RFS were performed as initial analyses. In univariate analysis, T4 stage (HR 2.52, 95\% Cl 1.40-4.55, $P=0.0021)$ and the location of the rectum (HR 2.19, 95\% $\mathrm{Cl} 1.32-3.63, P=0.002$ ) were identified as significant risk factors affecting the RFS (Table 2). In multivariate analysis, four factors were identified as significant risk factors affecting the RFS: the number of lymph node dissections less than 12 (HR 1.88, 95\% $\mathrm{Cl} 1.02-3.45, P=0.0412$ ), the location of the rectum (HR 2.13,95\% $\mathrm{Cl} 1.31-3.47, P=0.0023)$, T4 stage (HR 2.70, 95\% Cl 1.42-5.12, $P=0.0023$ ), and BD3 (HR 1.95, 95\% Cl 1.03-3.7, $P=0.0396$ ) (Table 2).

Primary analysis to investigate the association between T4 stage and BD3 for recurrence

Table 3 shows the clinical characteristics of 257 patients based on T4 stage and BD3 grade as primary analysis. There were 214 patients with $\mathrm{BD} 3$ without T4 stage (BD3+/T4-), 21 with T4 stage without BD3 (BD3-/T4+), and 22 with both BD3 and T4 stage (BD3+/T4+). The numbers of patients treated with postoperative adjuvant chemotherapy $(P<0.001)$ and preoperative treatment $(P=0.0045)$ were significantly higher in the BD3+/T4+ group. In the BD3+/T4+ group, $9(40.9 \%)$ patients were administered adjuvant chemotherapy including 1 patient treated with doublet regimen, and the 5-year RFS rate with or without adjuvant chemotherapy was $76.2 \%$ and $59.3 \%$ (HR 1.99, 95\% $\mathrm{Cl} 0.38-10.36, \mathrm{p}=0.4024)$, respectively. 
The relationships of the combination of T4 stage and BD3 for the 5-year RFS rate is shown in Figure 2: 81.3\% (95\% Cl 74.7-86.6) of the BD3+/T4- group, 74.6\% (95\% Cl 51.6-89.0) of the BD3-/T4+ group, and $66.2 \%(95 \% \mathrm{Cl} 43.8-83.1)$ of the $\mathrm{BD} 3+/ \mathrm{T} 4+$ group, respectively. Furthermore, Cox regression analysis showed that there was a significant difference in the RFS between patients in the BD3+/T4- and those in $\mathrm{BD} 3+/ \mathrm{T} 4+$ groups (HR 3.08, 95\% Cl 1.47-6.45, $P=0.0079$ ). In colon cancer patients, the 5-year RFS rate was $84.9 \%(95 \% \mathrm{Cl} 76.7-90.1), 90.7 \%$ (95\% Cl 56.1-98.7), and $61.3 \%(95 \% \mathrm{Cl} 31.0-84.7)$ in the BD3+/T4-, BD3-/T4+, and BD3/T4 group, respectively $(P=0.0061)$.

\section{Discussion}

To our knowledge, this study is the first one to show the relationship between BD grade and T stage in patients with pStage II CRC as a prognostic value for recurrence. In the initial analysis, T4 stage and BD3 grade were identified as independent risk factors affecting RFS. Furthermore, the primary analysis demonstrated that the 5-year RFS rate in the BD3+/T4+ group was significantly lower than that in the BD3+/T4- group (66.2 vs. $74.6 \%)(P=0.0079)$. These results suggest that $\mathrm{pStage}$ II CRC patients who had both BD3 and T4 stage factors had a poorer prognosis than those with other high-risk factors.

A recent study reported that the tumor microenvironment at the invasive front, including the BD, played an important role in CRC outcomes. ${ }^{14}$ The intensity of BD grade and fibrotic immature stroma promotes dedifferentiation and dissemination of cancer cells and the first step of invasion. On the other hand, T stage is known to be a significant risk factor for recurrence in all cancer types. In the SACURA trial, BD3 (T4 rate: $26.8 \%$ ) was associated with a poorer prognosis than BD1 (T4 rate: $10.4 \%$ ) and BD2 (T4 rate: $16.0 \%$ ), although the BD3 group had a higher T4 rate. ${ }^{12}$ The prognosis in the BD3 group might be strongly influenced by the T4 stage in the SACURA trial. In the present study, we revealed that pStage II CRC patients with both BD3 and T4 factors had much poorer prognosis.

The 5-year RFS rate of $66.2 \%$ in the BD3+/T4+ group is similar to that of stage IIIC colon cancer. Moreover, in the BD3+/T4+ group, the recurrence rate was lower in the adjuvant chemotherapy group (HR $1.99,95 \% \mathrm{Cl} 0.38-10.36, P=0.4024)$, although the significant superiority of adjuvant chemotherapy was not shown. This might be due to the insufficient efficacy of adjuvant chemotherapy because only one out of nine patients was administered a doublet regimen including oxaliplatin. In the ACTS-CC 02 trial, the oxaliplatin-based regimen was more effective than UFT/leucovorin in advanced disease, such as stage IIIC and N2b. ${ }^{15}$ In addition, in the AHIEVE-2 trial, which compared the 3-month with 6-month CAPOX or mFOLFOX6 regimens as adjuvant treatment, the non-inferiority of 3-month treatments was not shown and the 3-year disease-free survival (DFS) rate inT4 stage patients was less favorable in the 3-month treatments than in the 6 -month treatments (3-month $76.2 \%$ vs. 6 month $79.7 \%$, HR 1.28 [0.68-2.43]). ${ }^{16}$ The 6-month oxaliplatin-based regimen might improve the prognosis in both BD3 and T4 stage patients. 
Recently, several predictive biomarkers for recurrence in stage II colon cancer have been examined. Stage II colon cancer patients with microsatellite instability-high (MSI-H) had a good prognosis, and

fluoropyrimidine-based adjuvant chemotherapy had no effect on DFS and OS. ${ }^{17}$ In the SACURA trial, MSI$\mathrm{H}$ was observed in $7.2 \%$, and the 5-year RFS rate in MSI-H patients was $92.9 \%$, which was better than that in non-MSI-H patients ( $\mathrm{HR} \mathrm{0.40,95 \%} \mathrm{Cl} \mathrm{0.17-0.98,} P=0.045) .{ }^{18}$ Moreover, there was no difference in the 5year RFS rate between the adjuvant chemotherapy group and surgery-alone group (94.3 vs. $91.7 \%)$. In stage II colon cancer with MSI-H, adjuvant chemotherapy might be unnecessary. However, MSI testing requires immunohistochemistry, expensive gene analyses, and clinical evidence supporting their use is still insufficient.

In addition, recently, circulating tumor DNA (ctDNA), which are short nucleic acid fragments thought to be released in the systemic circulation as a result of tumor cell apoptosis or necrosis, ${ }^{19,20}$ has emerged as a promising biomarker of disease status for metastatic cancer. ${ }^{21-23}$ Moreover, the detection of ctDNA after curative resection of stage II colon cancer might identify patients who are at the highest risk of recurrence and help inform adjuvant treatment decisions. Among the patients with positive ctDNA after curative resection, $79 \%$ experienced recurrence, whereas $9.8 \%$ experienced recurrence with negative ctDNA after curative resection (HR 18, 95\% $\mathrm{Cl} 7.9-40, P<0.00) .{ }^{24}$ Although ctDNA cannot be measured in routine clinical practice, ctDNA might be superior to other clinicopathological measures as prognostic factors.

In the initial analysis, the number of examined lymph nodes was identified as a risk factor affecting RFS in multivariate analysis. Although the number of dissected lymph nodes was considered sufficient when 12 or more lymph nodes had been examined pathologically, this number depends on the length of the dissection or the location of the right or left side. Therefore, definitive evidence as a prognostic factor has been lacking. Lymph or venous invasion, perineural invasion, and histologic type were not associated with statistically significant risk factors for recurrence in the present study. Indeed, in the SACURA trial, there were no relationships between these factors and prognosis. Furthermore, the frequency of these factors was much lower than that of other prognostic factors, although perforation or intestinal obstruction might be a poor prognostic factor in NCCN guidelines. ${ }^{3}$

Our study had several limitations. First, the present study had a retrospective design in a single institution. Second, the location of the rectum was a poor prognostic factor and $42 \%$ of the patients enrolled in this study had rectal cancer. However, in patients with only colon cancer excluding the rectum, the 5-year RFS rate in those with both BD3 and T4 was also shown to be significantly lower than those in the other two groups. Third, the pathologic results might have differed among pathologists because pathological evaluation including BD was not performed by determined pathologists. Fourth, the MSI, which is considered predictive in the NCCN guideline, ${ }^{3}$ was not examined in the present study.

In conclusion, this study indicated that patients with pStage II CRC with both BD3 and T4 had clearly poorer prognostic features than those with only BD3 or T4 factors. We believe that doublet adjuvant chemotherapy, including oxaliplatin, should be considered for patients with pStage II CRC with both BD3 and T4 stages. 


\section{Declarations}

Acknowledgements

The authors would like to thank the patients who participated in this study and their families as well as the staff members. We would like to thank Editage (www.editage.com) for English language editing.

\section{Author contributions}

Conception and design: H.K., T.Y., K.H. Collection of date: H.K, T.Y., Y. I., H.Y., M. A., T.M., T.T., F.S., M.G., H.H., W.O., M.Y., K.T., J.O., K.U. Date analysis: H.K., T.Y. Manuscript writing: H.K., T.Y. All authors read and approved the final manuscript.

\section{Competing interests}

The author(s) declare no competing interests.

\section{References}

1. Center for Cancer Control and Information Services, National Cancer Center. Projected cancer statistics, 2018. http://ganjoho.jp/en/public/statistics/short_pred.html; 2018. Accessed [03, July 2020].

2. Engstrom, P.F., et al. NCCN clinical practice guidelines in oncology: colon carcinoma. J. Natl. Compr. Canc. Netw. 7, 778-831 (2009).

3. Benson, A.B. III, et al. American Society of Clinical Oncology recommendations on adjuvant chemotherapy for stage II colon cancer. J. Clin. Oncol. 2216, 3408-3419 (2004).

4. Labianca, R., Nordlinger, B., Beretta, G.D., Brouquet, A., Cervantes, A. Primary colon cancer: ESMO clinical practice guidelines for diagnosis, adjuvant treatment and follow-up. Ann. Oncol. 21 (Suppl 5), v70-v77 (2010).

5. Quah, H.M., et al. Identification of patients with high-risk stage II colon cancer for adjuvant therapy. Dis Colon Rectum. 51, 503-507 (2008).

6. Babcock, B.D., et al. High-risk stage II colon cancer: Not all risks are created equal. Ann. Surg. Oncol. 25, 1980-1985 (2018).

7. Prall, F., Nizze, H., Barten, M. Tumour budding as prognostic factor in stage I/II colorectal carcinoma. Histopathology. 47, 17-24 (2005).

8. Ueno, H., Murphy, J., Jass, J., Mochizuki, H., Talbot, I.C. Tumor buddings an index to estimate the potential of aggressiveness in rectal cancer. Histopathology. 40, 127-132 (2002).

9. Van Wyk, H.C., et al. The relationship between tumour budding, the tumour microenvironment and survival in patients with primary operable colorectal cancer. Br. J. Cancer. 115, 156-163 (2016).

10. Lugli, A., et al. Recommendations for reporting tumor budding in colorectal cancer based on the International Tumor Budding Consensus Conference (ITBCC) 2016. Mod. Pathol. 30, 1299-1311 
(2017).

11. Union for International Cancer Control. TNM Classification of Malignant Tumours. 8th ed. (John Wiley \& Sons, 2017).

12. Matsuda, C., et al. A randomised-controlled trial of 1-year adjuvant chemotherapy with oral tegafururacil versus surgery alone in stage II colon cancer: SACURA Trial. Eur. J. Cancer. 96, 54-63 (2018).

13. Ueno, H., et al. Prospective multicenter study on the prognostic and predictive impact of tumor budding in stage II colon cancer: results from the SACURA Trial. J. Clin. Oncol. 37, 1886-1894 (2019).

14. van Wyk, H.C., et al. The relationship between tumor budding, tumor microenvironment, and survival in patients with primary operable colorectal cancer. Ann. Surg. Oncol. 13, 4397-4404 (2019).

15. Eiji, S., et al. S-1 and oxaliplatin versus tegafur-uracil and leucovorin as postoperative adjuvant chemotherapy in patients with high-risk stage III colon cancer (ACTS-CC 02): A randomized, openlabel, multicenter, phase III superiority trial. Clin. Colorectal Cancer. 1, 22-31 (2020).

16. Yamazaki, K., et al. Oxaliplatin-based adjuvant chemotherapy duration (3 versus 6 months) for highrisk stage II colon cancer: the randomized phase III ACHIEVE-2 trial. Ann. Oncol. 32, 77-84 (2021).

17. Des Guetz, G., et al. Does microsatellite instability predict the efficacy of adjuvant chemotherapy in colorectal cancer? A systematic review with meta-analysis. Eur. J. Cancer. 45, 1890-1896 (2009).

18. Ishikawa, T., et al. Prognostic impact of MSI and 18qLOH in stage II colon cancer: A prospective biomarker study in the SACURA trial. Ann Oncol. 29 (Suppl 8), viii174-viii175 (2018).

19. Canzoniero, J.V., Park, B.H. Use of cell free DNA in breast oncology. Biochim. Biophys. Acta. 1865, 266-274 (2016).

20. Bettegowda, C., et al. Detection of circulating tumor DNA in early- and late-stage human malignancies. Sci. Transl. Med. 6, 224ra24; 10.1126/scitransImed.3007094 (2014).

21. Calapre, L., Warburton, L., Millward, M., Ziman, M., Gray, E.S. Circulating tumour DNA (ctDNA) as a liquid biopsy for melanoma. Cancer Lett. 404, 62-69 (2017).

22. Wan, J.C.M., et al. Liquid biopsies come of age: towards implementation of circulating tumour DNA. Nat. Re.v Cancer. 17, 223-238 (2017).

23. Heitzer, E., Ulz, P., Geigl, J.B. Circulating tumor DNA as a liquid biopsy for cancer. Clin. Chem. 61, 112123 (2015).

24. Tie, J., et al. Circulating tumor DNA analysis detects minimal residual disease and predicts recurrence in patients with stage II colon cancer. Sci. Transl. Med. 8:346ra92;

10.1126/scitranslmed.aaf6219 (2016).

25. Aim MB, et al. American Joint Committee on Cancer (AJCC). Cancer Staging Manual. 8th ed. (Springer, 2017).

26. Watanabe, T., et al. Japanese Society for Cancer of the Colon and Rectum (JSCCR) guidelines 2016 for the treatment of colorectal cancer. Int. J. Clin. Oncol. 23, 1-34 (2018).

\section{Tables}


Table. 1 Patient characteristics

Page 10/16 


\begin{tabular}{|c|c|}
\hline & $N=448$ \\
\hline \multicolumn{2}{|l|}{ Age, years } \\
\hline Median (range) & $70(26-88)$ \\
\hline \multicolumn{2}{|l|}{ Sex } \\
\hline Male/female & $260(58.0) / 188(41.9)$ \\
\hline \multicolumn{2}{|l|}{ Tumor location } \\
\hline Colon/rectum & $259(57.8) / 188(42.0)$ \\
\hline \multicolumn{2}{|l|}{ Obstruction } \\
\hline$(+)$ & $11(2.4)$ \\
\hline \multicolumn{2}{|l|}{ Tumor perforation } \\
\hline$(+)$ & $2(0.4)$ \\
\hline \multicolumn{2}{|l|}{ Histologic type } \\
\hline Tub/Pap/Muc/Por/Scc & $400(89.3) / 11(2.4) / 21(4.7) / 9(2.0) / 1(0.2)$ \\
\hline \multicolumn{2}{|l|}{ Number of lymph nodes } \\
\hline \$12 & $65(14.5)$ \\
\hline \multicolumn{2}{|l|}{ T factor } \\
\hline T4 & $43(9.6)$ \\
\hline \multicolumn{2}{|l|}{ Lymphatic invasion } \\
\hline Positive & $351(70.3)$ \\
\hline \multicolumn{2}{|l|}{ Venous invasion } \\
\hline Positive & $312(69.6)$ \\
\hline \multicolumn{2}{|l|}{ Perineural invasion } \\
\hline Positive & $99(22.0)$ \\
\hline \multicolumn{2}{|l|}{ Tumor budding } \\
\hline BD1/BD2/BD3 & $122(27.2) / 70(15.6) / 236(52.7)$ \\
\hline \multicolumn{2}{|l|}{ CEA } \\
\hline$\nabla 5$ & $147(32.8)$ \\
\hline \multicolumn{2}{|l|}{ Adjuvant chemotherapy } \\
\hline$(+)$ & $56(12.5)$ \\
\hline
\end{tabular}




\begin{tabular}{|l} 
Preoperative treatment \\
Chemoradiotherapy/chemotherapy $39(8.7) / 36(8.0)$
\end{tabular}

Data are presented as number (\%) unless otherwise stated

BD, tumor budding; CEA, carcinoembryonic antigen

Table. 2 Univariate and multivariate analyses for recurrence-free survival 


\begin{tabular}{|c|c|c|c|c|}
\hline \multirow{3}{*}{ Parameter } & \multicolumn{2}{|l|}{ Univariate analysis } & \multicolumn{2}{|c|}{ Multivariate analysis } \\
\hline & Hazard ratio & $P$ value* & Hazard ratio & $P$ value* \\
\hline & $(95 \% \mathrm{Cl})$ & & $(95 \% \mathrm{Cl})$ & \\
\hline \multicolumn{5}{|c|}{ Number of lymph nodes } \\
\hline$\geq 12$ & 1 & & 1 & \\
\hline$<12$ & $1.63(0.90-2.93)$ & 0.1047 & $1.88(1.02-3.45)$ & 0.0412 \\
\hline \multicolumn{5}{|c|}{ Lymphatic/venous invasion } \\
\hline Negative & 1 & & 1 & \\
\hline Positive & $1.49(0.68-3.26)$ & 0.3163 & $1.81(0.71-4.60)$ & 0.2115 \\
\hline \multicolumn{5}{|l|}{ T factor } \\
\hline T3 & 1 & & 1 & \\
\hline T4 & $2.52(1.40-4.55)$ & 0.0021 & $2.70(1.42-5.12)$ & 0.0023 \\
\hline \multicolumn{5}{|c|}{ Tumor budding } \\
\hline BD1 & 1 & & 1 & \\
\hline BD2 & $1.22(0.52-2.86)$ & 0.6437 & $1.17(0.48-2.86)$ & 0.7260 \\
\hline BD3 & $1.72(0.93-3.22)$ & 0.0845 & $1.95(1.03-3.741)$ & 0.0396 \\
\hline \multicolumn{5}{|c|}{ Adjuvant chemotherapy } \\
\hline$(+)$ & 1 & & 1 & \\
\hline$(-)$ & $1.34(0.70-2.56)$ & 0.3699 & $1.00(0.47-2.13)$ & 0.9909 \\
\hline \multicolumn{5}{|c|}{ Histologic type } \\
\hline tub/par & 1 & & 1 & \\
\hline muc/por & $0.83(0.30-2.28)$ & 0.7179 & $0.99(0.33-2.96)$ & 0.9918 \\
\hline \multicolumn{5}{|c|}{ Perineural invasion } \\
\hline Negative & 1 & & 1 & \\
\hline Positive & $1.51(0.89-2.57)$ & 0.1263 & $1.43(0.82-2.49)$ & 0.1996 \\
\hline \multicolumn{5}{|l|}{ Location } \\
\hline Colon & 1 & & 1 & \\
\hline Rectum & $2.13(1.31-3.47)$ & 0.0023 & $2.19(1.32-3.63)$ & 0.0024 \\
\hline
\end{tabular}

$\mathrm{BD}$, tumor budding; $\mathrm{CEA} ; \mathrm{Cl}$, confidence interval 
* $P$ values $<0.05$ are statistically significant

Table 3. Patient characteristics based on BD3 grade and T4 stage

\begin{tabular}{|c|c|c|c|c|}
\hline & BD3 without T4 & T4 without BD3 & BD3 and T4 & $\begin{array}{l}P \\
\text { value* }\end{array}$ \\
\hline & $N=214$ & $N=21$ & $N=22$ & \\
\hline Age, years & & & & 0.4867 \\
\hline Median (range) & $70(35-87)$ & $70(35-87)$ & $70(41-78)$ & \\
\hline Sex & & & & 0.6851 \\
\hline Male/female & $\begin{array}{l}126(58.8) / 88 \\
(41.1)\end{array}$ & $13(61.2) / 8(38.1)$ & $\begin{array}{l}15(68.2) / 7 \\
(31.8)\end{array}$ & \\
\hline Location & & & & 0.2788 \\
\hline Colon/rectum & $\begin{array}{l}139(65.0) / 75 \\
(35.0)\end{array}$ & $12(57.2) / 9(42.8)$ & $\begin{array}{l}11(50.0) / 11 \\
(50.0)\end{array}$ & \\
\hline Number of lymph nodes & & & & 0.1911 \\
\hline$<12$ & $30(14.0)$ & $6(28.6)$ & $1(4.5)$ & \\
\hline T factor & & & & $<0.001$ \\
\hline $\mathrm{T} 4$ & 0 & $21(100)$ & $22(100)$ & \\
\hline $\begin{array}{l}\text { Lymphatic/venous } \\
\text { invasion }\end{array}$ & & & & 0.4379 \\
\hline Positive & $182(85.0)$ & $20(95.2)$ & 19 (86.3) & \\
\hline Tumor budding & & & & $<0.001$ \\
\hline BD1/BD2/BD3 & 0/0/214 (100) & $\begin{array}{l}10(47.6) / 9 \\
(42.9) / 0\end{array}$ & $0 / 0 / 22(100)$ & \\
\hline Perineural invasion & & & & 0.1302 \\
\hline Positive & $45(21.0)$ & $8(38.0)$ & $7(31.8)$ & \\
\hline CEA & & & & 0.0246 \\
\hline$>5$ & $151(70.5)$ & $9(42.8)$ & $13(59.1)$ & \\
\hline Adjuvant chemotherapy & & & & $<0.001$ \\
\hline$(+)$ & $11(5.1)$ & $4(19.0)$ & $9(40.9)$ & \\
\hline Preoperative treatment & & & & 0.0045 \\
\hline CT/CRT & $5(2.3) / 14(6.5)$ & $2(9.5) / 0$ & $4(18.2) / 4(18.2)$ & \\
\hline
\end{tabular}


$\mathrm{BD}$, tumor budding; $\mathrm{CEA}$, carcinoembryonic antigen; $\mathrm{CT}$, chemotherapy; $\mathrm{CRT}$, chemoradiotherapy

Data are presented as number (\%) unless otherwise stated

* $P$ values $<0.05$ are statistically significant

\section{Figures}

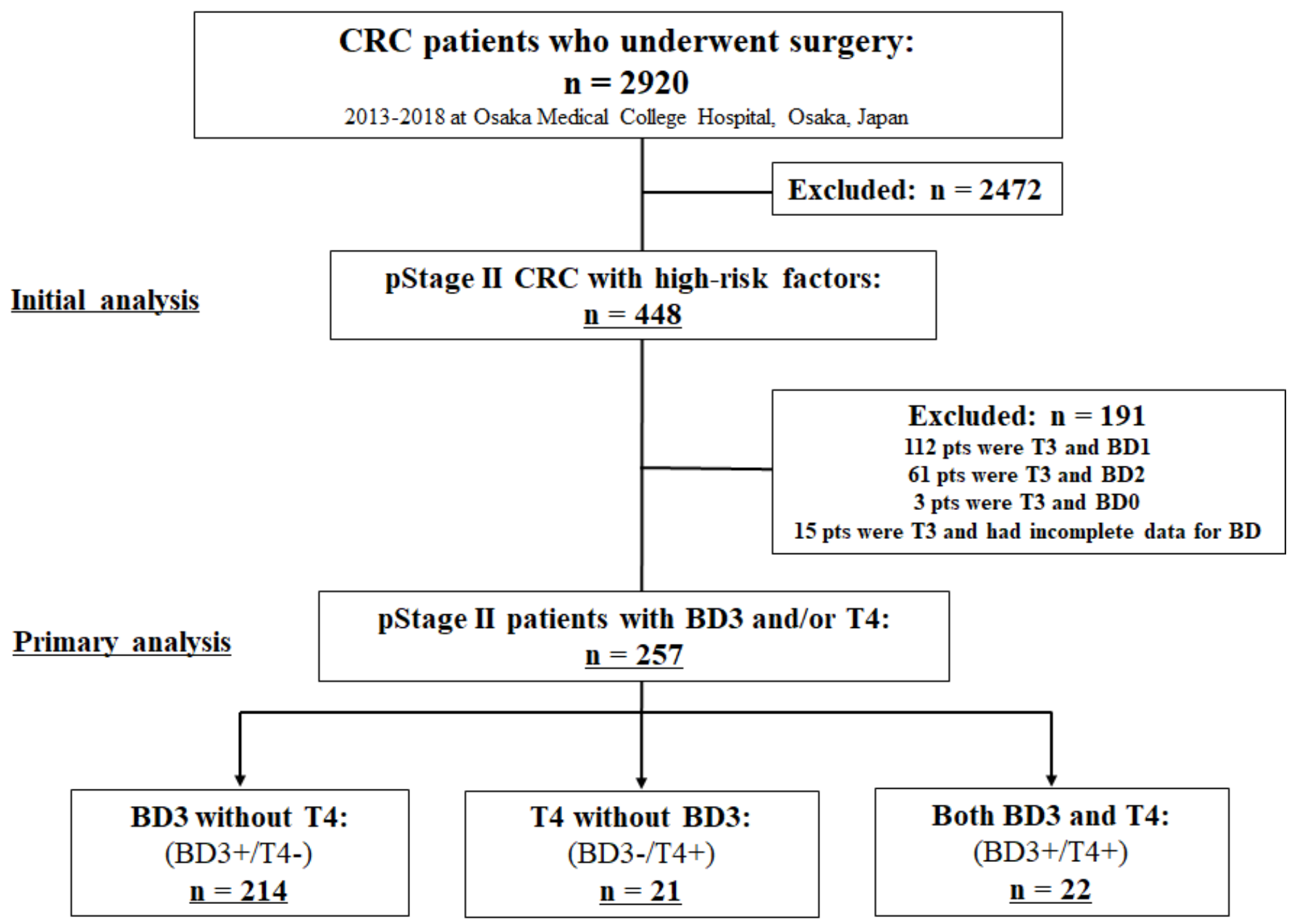

Figure 1

CONSORT diagram of this study. 


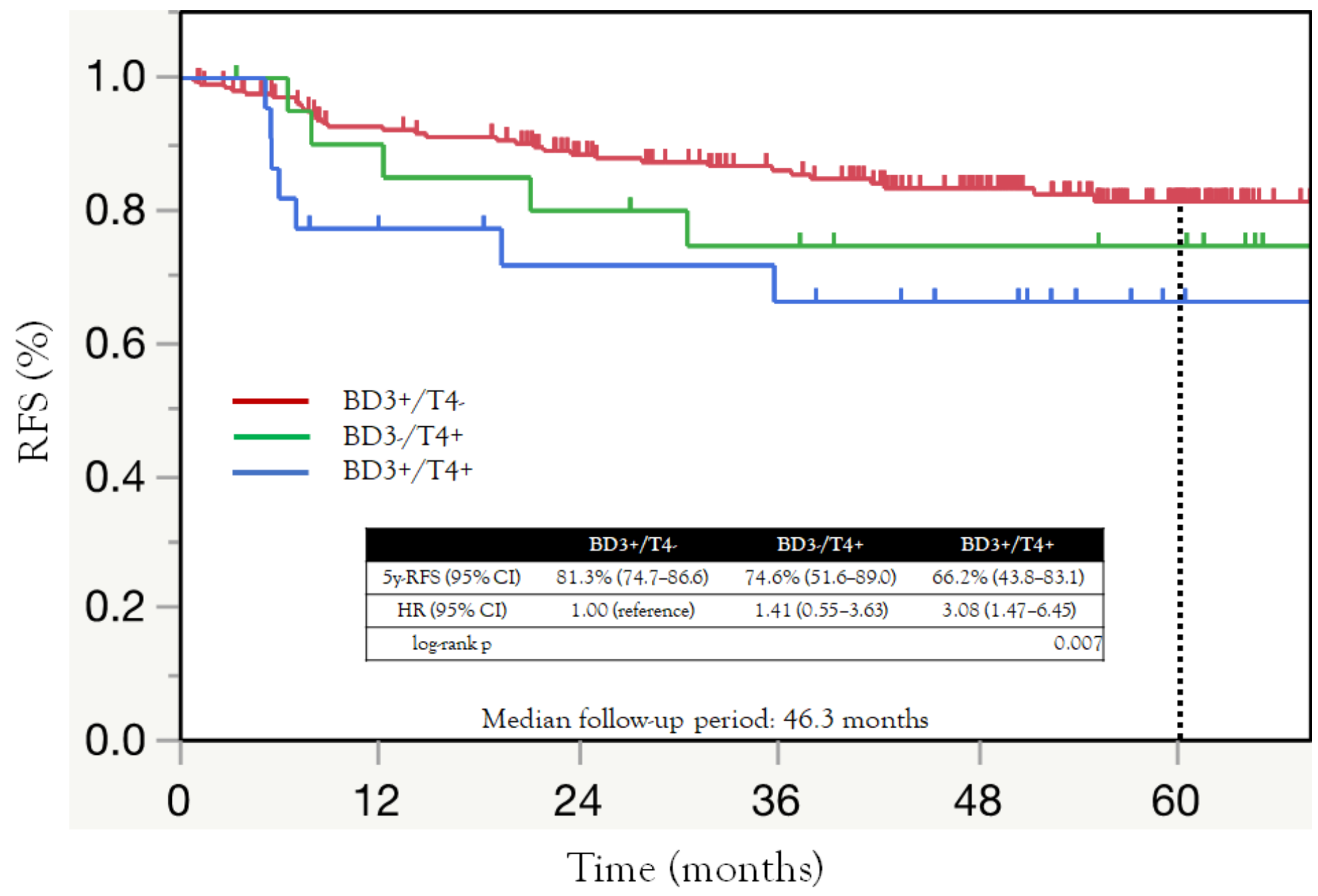

Figure 2

Kaplan-Meier estimates of recurrence-free survival based on BD3 and T4 stage. 